\title{
Effect of Stacking Fault Energy on the Grain Structure Evolution of FCC Metals During Friction Stir Welding
}

\author{
Xiaochao Liu $^{1,2} \cdot$ Yufeng Sun ${ }^{1,3} \cdot$ Tomoya Nagira $^{1} \cdot$ Kohsaku Ushioda $^{1} \cdot$ Hidetoshi Fujii ${ }^{1}$
}

Received: 13 February 2020 / Revised: 7 March 2020 / Published online: 13 May 2020

(c) The Chinese Society for Metals (CSM) and Springer-Verlag GmbH Germany, part of Springer Nature 2020

\begin{abstract}
The effect of stacking fault energy (SFE) on the grain structure evolution of face-centered cubic metals during friction stir welding was investigated by using pure aluminum, pure copper and $\mathrm{Cu}-30 \mathrm{Zn}$ alloy as experiment materials. Tool "stop action" and rapid cooling were employed to "freeze" the microstructure of the flowing materials around the tool. Marker materials were used to show the streamline of the material flow. The microstructures of the three materials at different welding stages were contrastively studied by the electron backscatter diffraction technique. The results show that at the material flow stage, as the SFE decreases, the grain structure evolution changes from the continuous dynamic recrystallization to discontinuous dynamic recrystallization, and further to the dynamic equilibrium between the annealing twinning due to thermally activated grain boundary migration and the twin destruction during the plastic deformation. Owing to different grain structure evolution mechanisms, the grain structure at the end of the material flow is greatly different. Especially in copper, a lot of dislocations remain, which gives rise to the static recrystallization occurring during the subsequent cooling stage.
\end{abstract}

Keywords Stacking fault energy $\cdot$ Friction stir welding $\cdot$ Microstructure evolution $\cdot$ Face-centered cubic (FCC) metal . Electron backscattered diffraction (EBSD) $\cdot$ Recrystallization

\section{Introduction}

Friction stir welding (FSW) is a solid-state joining technique invented in 1991 by TWI [1]. It has great flexibility for welding various materials and structures and thus bears great expectations in the field of solid-state joining [2]. In FSW, a non-consumable rotating tool is plunged into the workpiece and moved along the joint line. The friction between the tool and the workpiece gives rise to heat softening of the material and subsequent plastic flow, forming a sound joint

Available online at http://link.springer.com/journal/40195.

Xiaochao Liu

xcliu@nwpu.edu.cn

Hidetoshi Fujii

fujii@jwri.osaka-u.ac.jp

1 Joining and Welding Research Institute, Osaka University, 11-1 Mihogaoka, Ibaraki, Osaka 567-0047, Japan

2 School of Materials Science and Engineering, Northwestern Polytechnical University, Xi' an 710072, China

3 School of Materials Science and Engineering, Zhengzhou University, Zhengzhou 450001, China
$[3,4]$. During this process, complex physical metallurgical phenomena including plastic deformation [5], recovery [6], recrystallization [7] and grain growth [8] occur. These microstructural evolution mechanisms determine the final microstructure and mechanical properties of the joints [9]. In order to control the microstructure of the joints, it is of importance to elucidate the mechanisms of microstructure evolution during the FSW.

FSW process is essentially plastic deformation at elevated temperature and subsequent annealing during the cooling stage [10]. The changes in microstructure during this process are highly dependent on the properties of the materials crystal structure. For example, different lattice structures have different slip systems. Different slip systems play different roles at different deformation temperatures, strain and strain rates, which result in different grain structures and textures. The materials which have the same lattice structure may also have different stacking fault energy (SFE), which has large influence on the dislocation motion during the plastic deformation [11] and on the annealing twin formation during the grain boundary migration [12]. Therefore, the clarification of the microstructure evolution during the FSW of materials with different lattice structures and SFE is necessary. 
There have been many studies trying to reveal the microstructure evolution during the FSW of metals with different lattice structures. Prangnell and Heason studied the grain structure formation during the FSW of aluminum alloy using a "stop action technique" [13]. Mironov et al. investigated the grain structure development during the FSW of pure iron [14] and pure titanium [15]. Wang et al. summarized the microstructure evolution of magnesium alloys during the FSW [16]. The most studied materials are face-centered cubic (FCC) metals. It has been well accepted that the continuous dynamic recrystallization (CDRX) dominates the grain structure development during the FSW of aluminum and its alloys [17-19], which have relatively high SFE. However, Huang et al. [20] reported an atypical grain coarsening phenomenon existing in the stir zone of AA6082-T6 aluminum alloy, which only occurred at certain conditions, such as extremely high plastic strain and strain rate by friction stir welding, and an elevated temperature slightly above 0.5 melting point to inhibit the DRX refinement under higher temperature and shear-induced refinement under lower temperature. If the SFE is relatively low, such as pure copper, the CDRX still dominates the grain structure evolution when the welding peak temperature is lower than $\sim 0.5 T_{\mathrm{m}}$ (where $T_{\mathrm{m}}$ is the melting point), but the grain structure development at temperatures above $\sim 0.5 T_{\mathrm{m}}$ is dominated by discontinuous dynamic recrystallization (DDRX) [21]. As the SFE decreases further, such as $\mathrm{Cu}-30 \mathrm{Zn}$ brass, the DDRX dominates the grain structure development at all the ranges of temperature [22]. However, these results were drawn by the observation of microstructure on the weld transverse cross section or longitudinal cross section or around the keyhole. They are not along the true material flow path, resulting in that the dynamic microstructure evolution during the material flow cannot be observed directly. Liu et al. [23] pointed out that a rotational flow zone exists around the tool in their recent study. The material in the rotation flow zone travels with the tool in the welding direction for a long distance and deposits in the weld surface finally. This means that the material in the rotational flow zone does not affect the final weld microstructure. Unfortunately, many studies did not pay attention to this issue. Recently, Liu et al. [24-26] have developed a quasi-in situ method to evaluate the dynamic development of grain structure and texture during the FSW. In this method, a foil which is similar to the base material is inserted into the butting surface of the workpieces as marker material to show the streamline of material flow. Tool "stop action" and rapid cooling are employed to "freeze" the microstructure of the flowing material around the tool. Then, the microstructure evolution is investigated along the streamline. In this way, the microstructure development during the welding process can be captured. By using this method, Liu et al. investigated the grain structure and texture development during the FSW of pure aluminum
[27], pure copper [25, 26], pure iron [28] and $\mathrm{Cu}-30 \mathrm{Zn}$ brass [29], respectively. However, the effect of SFE on the grain structure development at different welding stages has not yet been summarized.

In this study, the FCC metals, pure aluminum, pure copper and $\mathrm{Cu}-30 \mathrm{Zn}$, with different SFE (correspondingly $166 \mathrm{~mJ} \mathrm{~m}^{-2}, 78 \mathrm{~mJ} \mathrm{~m}^{-2}$ and $20 \mathrm{~mJ} \mathrm{~m}^{-2}$ ) [12] were used as the experimental materials to investigate the effect of SFE on the grain structure evolution during the FSW.

\section{Experimental}

The materials used in this study were commercial Al1050H24, C1100 (1/4H) and C2600 (1/2H) in JIS (Japanese Industrial Standards). They were supplied as 3-mm-thick sheets and then machined into small workpieces with the dimensions of $200 \mathrm{~mm}$ in length and $50 \mathrm{~mm}$ in width by shearing and milling. Figure 1a shows the welding procedure, which has been documented in detail in our previous papers [25]. Two workpieces were butted, and a foil was inserted into the faying surface as marker material. The tool was plunged into the workpieces at the starting point where no marker material existed, and then moved into the part where marker material existed. This is to avoid that the marker material was involved into the rotational flow zone. At the end of the workpieces, the tool "stop action" was operated, i.e., the tool lost power suddenly and was pulled out instantaneously. During the whole process, a synchronous rapid cooling using liquid $\mathrm{CO}_{2}$ as cooling medium was adopted to "freeze" the microstructure. However, it is worth mentioning that this synchronous rapid cooling only cooled the weld exposed to the liquid $\mathrm{CO}_{2}$. The lower region of the weld still underwent a short-time annealing [25, 29]. This is because the material flow terminated immediately once the material migrated to the rear side of the tool pin, but the liquid $\mathrm{CO}_{2}$ was still shielded by the shoulder. The material here could not be cooled immediately, but entered an annealing state. Only after the tool shoulder fully passed, the weld could be cooled completely. Nevertheless, the microstructure near the keyhole can be "frozen" during the tool "stop action" because the keyhole was fully exposed to the liquid $\mathrm{CO}_{2}$ after the tool was pulled out.

The used tool was a tungsten carbide tool containing a concave shoulder $\left(10^{\circ}\right)$ and a cylindrical probe of $2.8 \mathrm{~mm}$ in length with a smooth surface. The diameters of the shoulder and the probe were 12 and $4 \mathrm{~mm}$, respectively. The tool tilt angle was $3^{\circ}$. The welding parameters (tool rotating speed-welding speed) for the pure aluminum, pure copper and $\mathrm{Cu}-30 \mathrm{Zn}$ were $800 \mathrm{rpm}-200 \mathrm{~mm} / \mathrm{min}$, $800 \mathrm{rpm}-150 \mathrm{~mm} / \mathrm{min}$ and $800 \mathrm{rpm}-150 \mathrm{~mm} / \mathrm{min}$, respectively. They roughly correspond to a welding peak temperature of $0.6 T_{\mathrm{m}}-0.7 T_{\mathrm{m}}$ for each material $[21,22,30]$, 

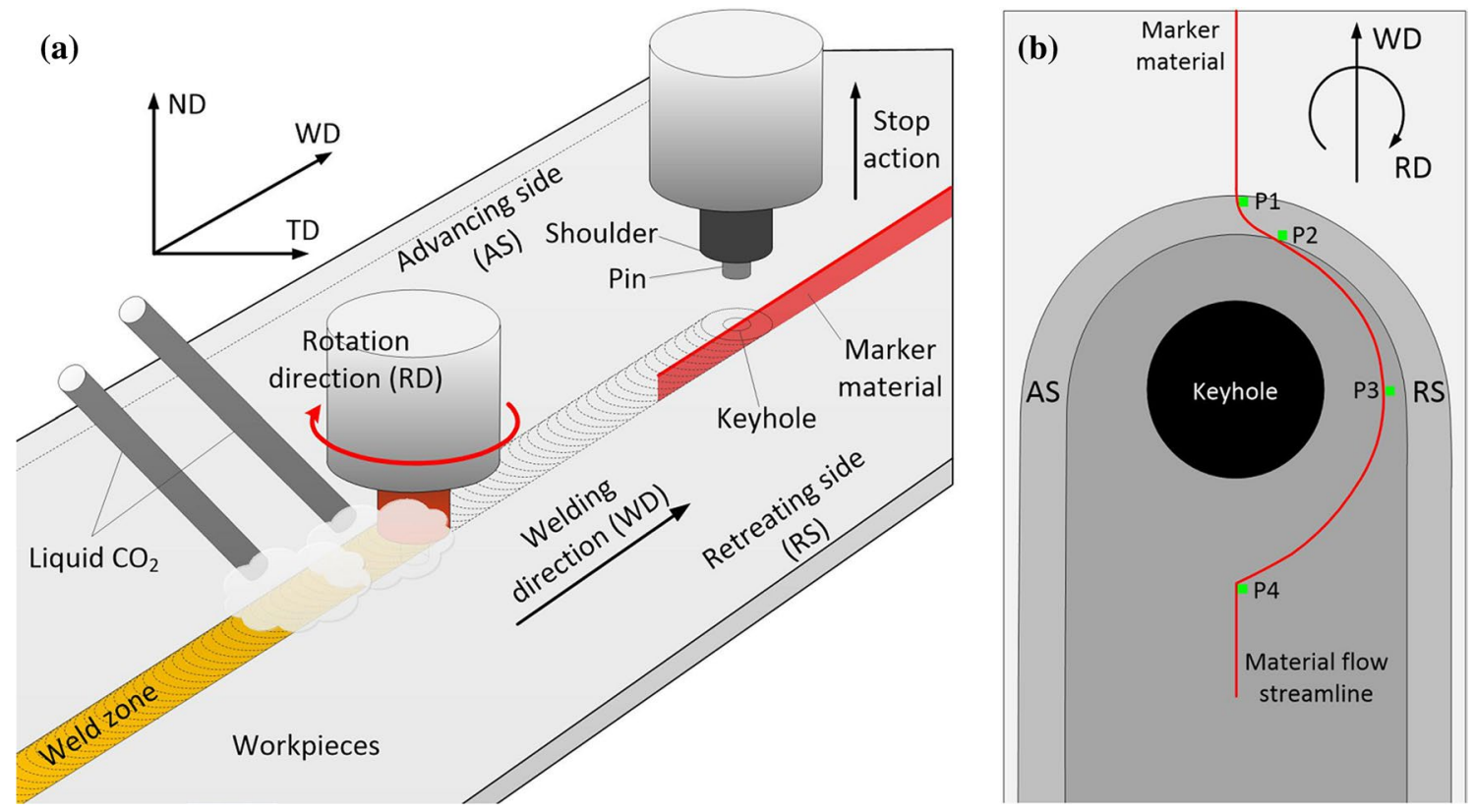

Fig. 1 Schematic of the experiment: a welding procedure; $\mathbf{b}$ EBSD measured positions

which stands for general welding conditions to obtain good weld formation and joint mechanical properties. The schematic of the electron backscattered diffraction (EBSD) measured positions is shown in Fig. 1b. On the keyhole horizontal cross section, the positions where the plastic deformation was beginning, the recrystallization was beginning, the material was high-speed flowing, and the material flow was terminated were selected as the EBSD measured points and were recorded as P1-P4 (Fig. 1b), denoting different welding stages. The microstructures after a short-time annealing were also characterized by the EBSD technique, to simulate the microstructure evolution during the cooling stage of the FSW. The details of the annealing conditions refer to our previous studies $[25,27,29]$.

For the EBSD measurement, the specimen preparation for the three materials refers to Refs. [25, 27, 29], respectively. A JEM-7001FA field emission scanning electron microscope (FE-SEM) operating at $15 \mathrm{kV}$ with the TSL OIM ${ }^{\mathrm{TM}}$ system was used. The step size of the EBSD scanning ranged from 0.25 to $1 \mu \mathrm{m}$, depending on the grain size. The low-angle boundary (LAB) and high-angle boundary (HAB) were distinguished by misorientation angle $2^{\circ}-15^{\circ}$ and $>15^{\circ}$, respectively. The $\Sigma 3$ twin boundary (TB) was determined by the $<111>/ 60^{\circ}$ axis/angle pair with a tolerance of $5^{\circ}$. The grains were identified by a continuous boundary (misorientation angle $>2^{\circ}$ ) in the EBSD maps. The grain size was quantified by determining the area of each grain in the EBSD map and calculating its circle equivalent diameter, which can be directly read from the OIM analysis software.

\section{Results}

Figure 2 shows the microstructural features of the base materials with different SFE. Since the supplied materials were in a work hardened state, a lot of LABs existed in the grains of the base materials. In the high SFE metal, pure aluminum, the LAB fraction was as much as $70.9 \%$. In the mid-SFE metal, pure copper, the LAB fraction decreased to $60.1 \%$, but there existed $11.7 \% \sum 3 \mathrm{~TB}$. In the low SFE metal, $\mathrm{Cu}-30 \mathrm{Zn}$, the LAB fraction was only $40.8 \%$, but the fraction of $\sum 3$ TB increased to $21.3 \%$. The $\sum 3$ TBs in the mid- and low SFE metals were actually residual annealing TBs, which remained because the base materials were produced by first annealing and then partially cold working. The morphologies of the LAB in different SFE metals were greatly different. In the high SFE metal, the LABs were continuous and uniformly distributed, but did not form a mass of subgrains, as shown in Fig. 2a. In the mid-SFE metal, they were agglomerated in the grain interior (Fig. 2b). In the low SFE metal, they gathered along with the HABs (Fig. 2c).

Figure 3 shows the microstructural features of metals with different SFE at the deformation beginning position (P1) during the FSW. The EBSD grain boundary maps are shown in Fig. $3 a_{1}-c_{1}$. The corresponding kernel average misorientation (KAM) maps are shown in Fig. $3 a_{2}-c_{2}$. The $\mathrm{KAM}$ value is a measure of the average misorientation of the neighboring orientation points relative to a given center orientation point. Here the first neighbor shell was considered. Misorientations $>5^{\circ}$ are discarded to avoid an artificial influence from adjacent grain boundaries. It is usually 




Fig. 2 Microstructural features of the base materials: a-c EBSD grain boundary maps; a pure aluminum; $\mathbf{b}$ pure copper; $\mathbf{c}$ Cu-30Zn
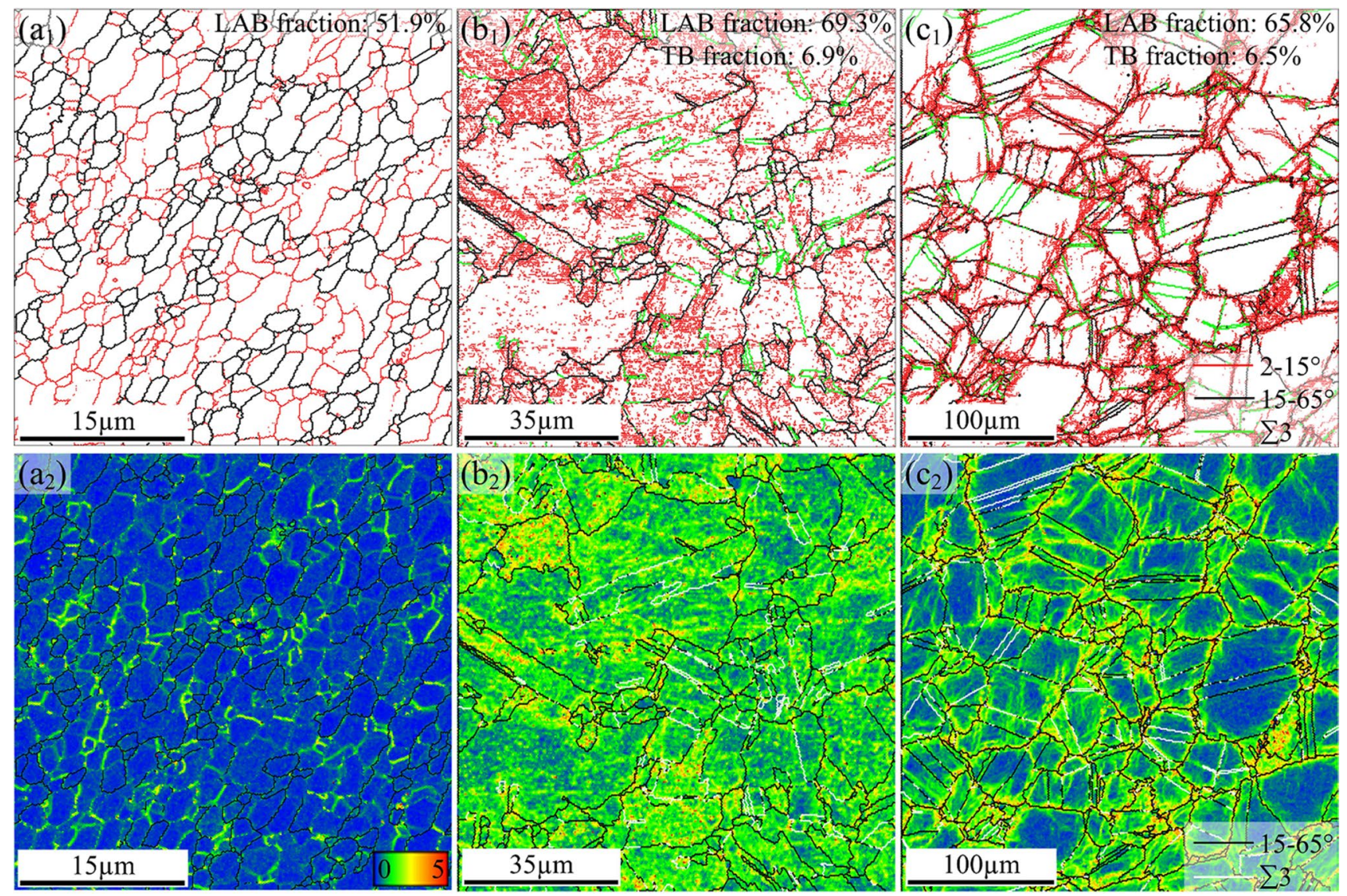

Fig. 3 Microstructural features at the deformation beginning position (P1): $\mathbf{a}_{\mathbf{1}}-\mathbf{c}_{\mathbf{1}}$ EBSD grain boundary maps; $\mathbf{a}_{\mathbf{2}}-\mathbf{c}_{\mathbf{2}}$ the corresponding KAM maps; $\mathbf{a}_{\mathbf{1}, 2}$ pure aluminum; $\mathbf{b}_{\mathbf{1 , 2}}$ pure copper; $\mathbf{c}_{\mathbf{1 , 2}} \mathrm{Cu}-30 \mathrm{Zn}$

used to characterize the dislocation density qualitatively. In the high SFE metal, pure aluminum, a lot of equiaxed subgrains formed, as shown in Fig. $3 \mathrm{a}_{1}$. Also, the LAB fraction decreased to $51.9 \%$. It means that some of the LABs had transformed into the HABs during the deformation.
The KAM map in Fig. $3 \mathrm{a}_{2}$ shows that the average misorientation was very small, which means that the dislocation density was relatively low. The processes dominating this microstructure evolution are known as the dynamic recovery (DRV) [31, 32] and the continuous dynamic recrystallization 
(CDRX) [33, 34]. In the mid-SFE metal, pure copper, the LAB fraction increased to $69.3 \%$, and the $\sum 3 \mathrm{~TB}$ fraction decreased to $6.9 \%$, as shown in Fig. $3 b_{1}$. This means that more and more LABs formed and partial TBs lost the twin relationship with the strain increasing. The LABs were still discontinued. The KAM map in Fig. $3 b_{2}$ shows that the dislocation distribution was nearly uniform and the dislocation density was very high. This means that the dislocations produced by plastic deformation were almost remained and no DRV and CDRX occurred during the deformation beginning stage. For the low SFE metal, $\mathrm{Cu}-30 \mathrm{Zn}$, as the strain increased, more and more LABs formed and gathered along with the original HABs. At the same time, some $\sum 3$ TBs accommodated the plastic deformation and transformed into general HABs. The LAB fraction increased to $65.8 \%$, and the $\sum 3 \mathrm{~TB}$ fraction decreased to $6.5 \%$, as shown in Fig. $3 \mathrm{c}_{1}$. The KAM map in Fig. $3 c_{2}$ shows that the dislocation distribution was very nonuniform. The dislocation density near the original HABs was very high, but in the grain interior was relatively low.

The microstructural features of the metals with different $\mathrm{SFE}$ at the recrystallization beginning position (P2) during the FSW are shown in Fig. 4. For the high SFE metal, as shown in Fig. $4 a_{1}$ and $a_{2}$, the LAB fraction decreased from 51.9 to $27.7 \%$. The dislocation density was still very low. Most of the LABs had transformed into HABs at this stage. This means that the process of CDRX had fully developed. This agrees well with the results reported by the study of Mironov et al. on FSW of pure aluminum [35]. In the midSFE metal, the LAB fraction decreased from 69.3 to $55.3 \%$. There existed some grains in which no LABs can be seen, as shown in Fig. $4 b_{1}$. The KAM map in Fig. $4 b_{2}$ shows that the dislocation density in these grains was also very low. These grains are known as recrystallized grains which were developed by discontinuous dynamic recrystallization (DDRX) [36]. This accords with the view of Mironov et al. [21], but does not agree with the study of Heidarzadeh et al. [37]. The latter thought that the CDRX and static recovery are the active mechanisms during the FSW of pure copper. The $\sum 3$ TB fraction was only $6.4 \%$, which only can be found in the grains without LABs. This indicates that the $\sum 3 \mathrm{TBs}$ were formed during the HABs migration, which is known as annealing twin [38]. In the low SFE metal, owing to the gathered dislocations near the original HABs, the recrystallized
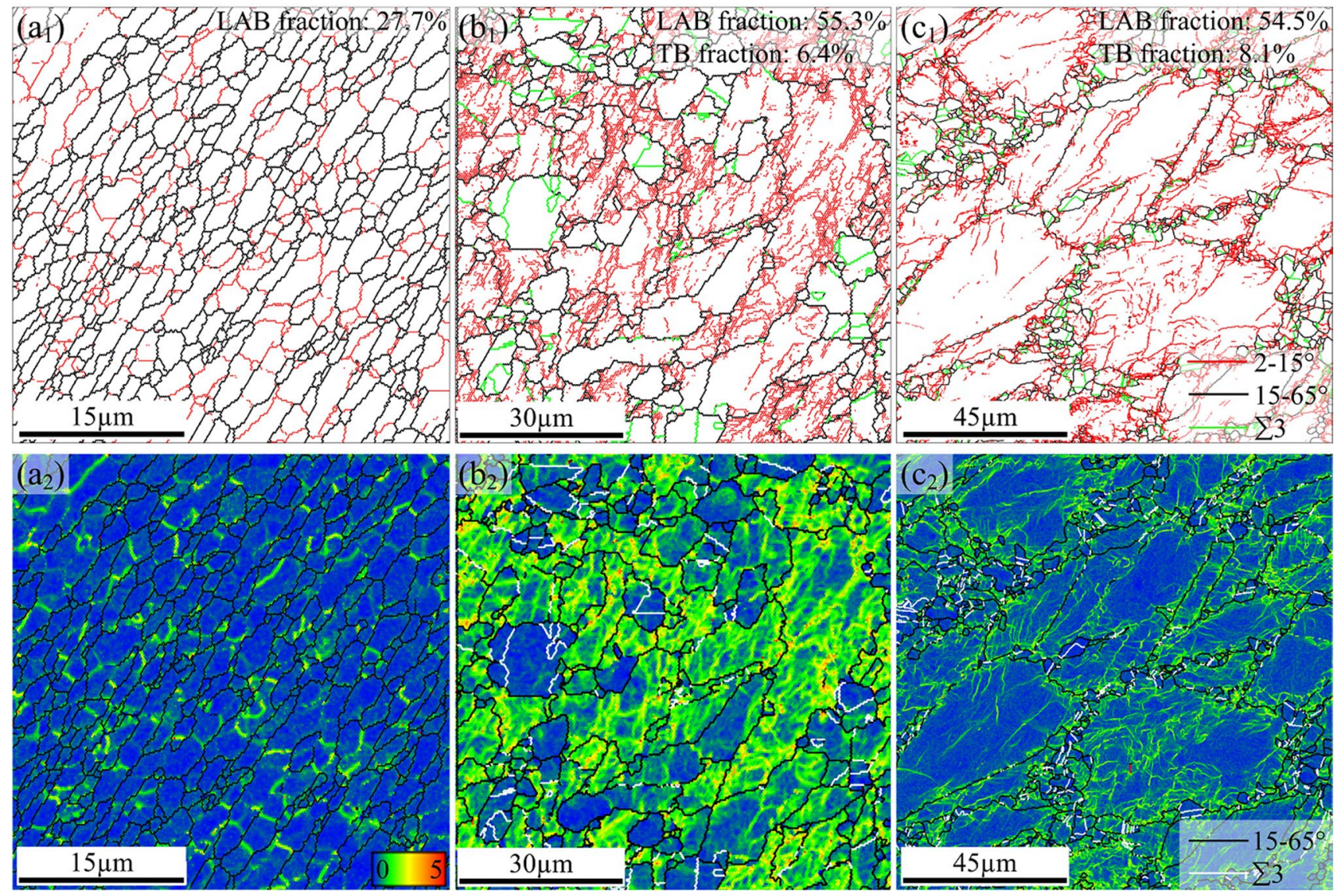

Fig. 4 Microstructural features at the recrystallization beginning position (P2): $\mathbf{a}_{1}-\mathbf{c}_{\mathbf{1}}$ EBSD grain boundary maps; $\mathbf{a}_{2}-\mathbf{c}_{2}$ the corresponding KAM maps; $\mathbf{a}_{\mathbf{1}, 2}$ pure aluminum; $\mathbf{b}_{\mathbf{1}, 2}$ pure copper; $\mathbf{c}_{\mathbf{1 , 2}} \mathrm{Cu}-30 \mathrm{Zn}$ 
grains were first nucleated along the original HABs, forming the typical necklace-like structure, as shown in Fig. $4 \mathrm{c}_{1}$ and $c_{2}$. This is different from that in the mid-SFE metal, in which the recrystallized grains were randomly distributed due to that the dislocations were accumulated uniformly. The similar phenomenon was also reported by the study of Mironov et al. on the FSW of $\mathrm{Cu}-30 \mathrm{Zn}$ [22]. The $\sum 3$ TBs only existed in the recrystallized grain. As mentioned above, they were developed during the HABs migration and thus were also classified to annealing twin. The recrystallization mechanism in the low SFE metal also belongs to DDRX. In addition, it is worth mentioning that in the mid- and low SFE metals, the morphology of the LABs at the recrystallization beginning stage was remarkably different from that at the deformation beginning stage. Although they could not evolve into subgrains just like that in high SFE metal, they gradually became continuous. This means that the DRV also occurred at this stage due to the increasing deformation temperature. The study of Heidarzadeh et al. also mentioned this phenomenon [37].

Figure 5 shows the microstructural features of the metals with different SFE at the material high-speed flowing stage during the FSW. For the high SFE metal, as shown in Fig. $5 a_{1}$ and $a_{2}$, the grain structures were similar to that at the recrystallization beginning stage (Fig. $4 a_{1}$ ), which consisted of equiaxed grains and included $30 \%$ LABs. The KAM map in Fig. $5 \mathrm{a}_{2}$ shows that the dislocation density was also very low. This indicated that the CDRX dominated the grain structure evolution during the material high-speed flowing stage [29]. As for the mid-SFE metal, the LAB fraction decreased to $35.7 \%$. At the same time, the average grain size also decreased obviously. The grains structures consisted of recrystallized grains with $\sum 3$ TBs inside and deformed grains with LABs inside, as shown in Fig. $5 b_{1}$. In the recrystallized grains, the dislocation density was very low, but in the deformed grains, the dislocation density was very high, as shown in Fig. $5 b_{2}$. These features show that the grain refinement during the material flow of mid-SFE metal was predominated by the DDRX [25]. However, in the low SFE metal, the grain structure was greatly different from that in the high and mid-SFE metals. In the low SFE metal, the LAB fraction was only 7.9\%. However, the $\sum 3$ TB fraction was as much as $33.5 \%$, as shown in Fig. $5 \mathrm{c}_{1}$. The KAM map in Fig. $5 c_{2}$ shows that the dislocation density was quite low
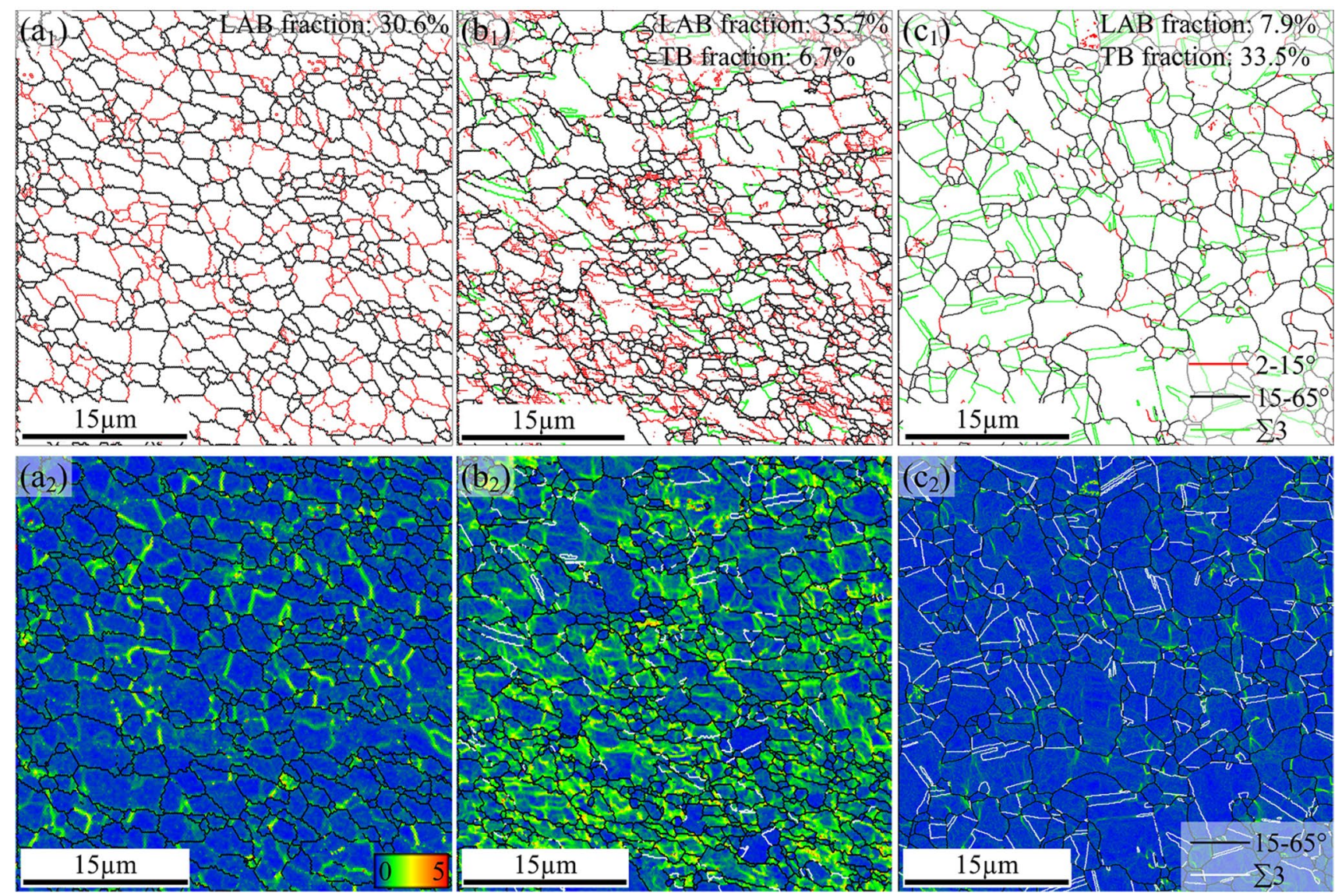

Fig. 5 Microstructural features at the high-speed flowing stage (P3): $\mathbf{a}_{\mathbf{1}}-\mathbf{c}_{\mathbf{1}}$ EBSD grain boundary maps; $\mathbf{a}_{\mathbf{2}}-\mathbf{c}_{\mathbf{2}}$ the corresponding KAM maps; $\mathbf{a}_{\mathbf{1}, 2}$ pure aluminum; $\mathbf{b}_{\mathbf{1}, \mathbf{2}}$ pure copper; $\mathbf{c}_{\mathbf{1 , 2}} \mathrm{Cu}-30 \mathrm{Zn}$ 
and even lower than that in the high SFE metal. This means that an unusual mechanism predominated the grain structure evolution of low SFE metal at the high-speed flowing stage during the FSW.

In order to reveal the mechanism of the grain structure evolution of low SFE metal at the high-speed flowing stage during the FSW, some details of the grain structure evolution are shown in Fig. 6. Figure 6a shows the EBSD image quality (IQ) map of the grain structure at the high-speed flowing stage. It can be seen that the image quality was very high. This indicates that distortion of the EBSD sample surface was very small, which was coherent with the result shown by the KAM map in Fig. $5 c_{2}$, i.e., the dislocation density was quite low. Figure $6 \mathrm{~b}$ shows the statistic misorientation angle distribution of the grain boundaries at the high-speed flowing stage. The dominant feature was that in the HAB range $\left(>15^{\circ}\right)$, the grain boundary fractions decreased gradually as the misorientation angle decreased. In the LAB range $\left(2^{\circ}-15^{\circ}\right)$, the grain boundary fraction was very low. This is quite different from that in the high SFE metals [27] or midSFE metals [39]. The maximum grain boundary fraction located near $60^{\circ}$, which corresponded to the $\sum 3 \mathrm{~TB}$. This was in accord with the statistic result shown in Fig. $5 c_{1}$. The $\sum 3$ TBs in Fig. 6a present the features of the annealing twin boundary. An example is shown in Fig. 6c, which is a magnification of the region $\mathbf{c}$ selected in Fig. 6a. Inside the grain, three $\sum 3$ TBs formed. Two of them even did not change the grain orientation. These $\sum 3 \mathrm{TBs}$ were formed due to a stacking fault occurring during the HABs migration. Owing to that there is no obvious dislocation density difference between the two sides of the HABs, this kind of HABs migration is regarded as thermally activated grain boundary migration [12]. At the same time, the phenomenon of the twin destruction also could be seen here and there. An example is shown in Fig. 6d, which is a magnification of the region d selected in Fig. 6a. The $\sum 3$ TB had partially transformed into a general HAB due to the loss of twinning relationship, as pointed out by the white arrow in the IPF map. The corresponding KAM map shows that the dislocation density along the $\sum 3$ TBs was obviously higher than that in other regions. These results indicated that the $\sum 3$ TBs absorbed the dislocations produced by the
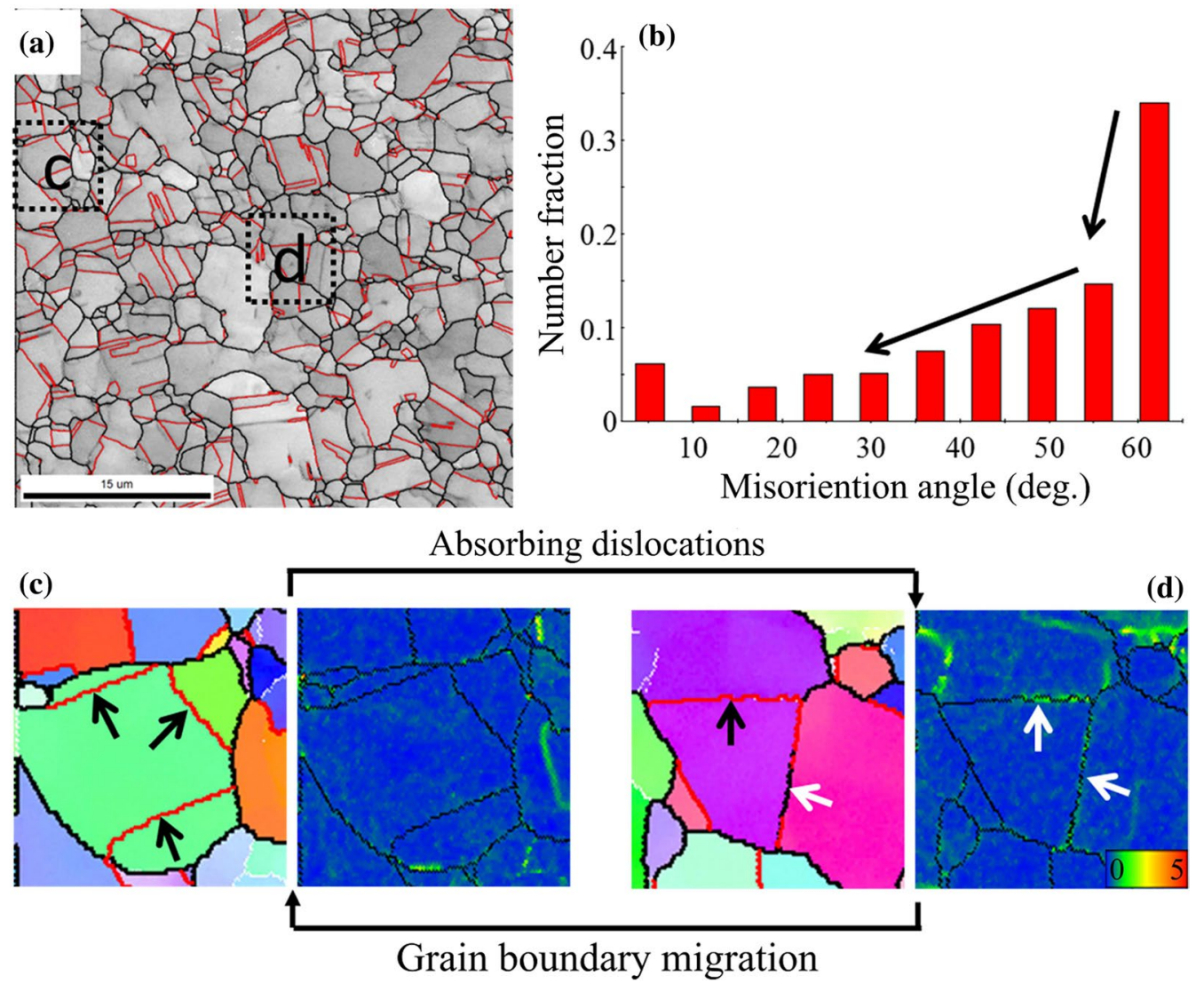

Fig. 6 Dynamic equilibrium between the annealing twinning due to the thermally activated grain boundary migration and the twin destruction due to the continuous deformation during the material flow in the FSW of $\mathrm{Cu}-30 \mathrm{Zn}$ : a EBSD IQ map; $\mathbf{b}$ the corresponding misorientation angle distribution; $\mathbf{c}$ the IPF and KAM maps of the region $\mathbf{c}$ selected in $\mathbf{a}$; $\mathbf{d}$ the IPF and KAM maps of the region $\mathbf{d}$ selected in $\mathbf{a}$. Note: in the IQ and IPF maps, the black line donates HABs and the red line denotes $\sum 3$ TBs 
plastic deformation and transformed into general HABs. This agreed well with that the grain boundary fractions gradually decreased as the misorientation angle decreased. Then, the general HABs migrated due to the thermal activation. During the HABs migration, the $\sum 3 \mathrm{TBs}$ formed as a result of the incidental occurrence of a stacking fault for such a low SFE metal. This explained why the $\sum 3$ TB fraction was maximum in the misorientation angle distribution. Thus, the grain structure evolution of the low SFE metal at the highspeed flowing stage during the FSW can be described as the dynamic equilibrium between the annealing twinning due to the thermally activated grain boundary migration and the twin destruction due to the continuous deformation during the material flow [29]. Also, the similar mechanism was recently found during the FSW of pure silver by the authors' group [40]. This mechanism has never been discovered by the previous studies [21, 22, 37].

Figure 7 shows the microstructure features of the metals with different SFE at the material flow ending position (P4 in Fig. 1b). For the high SFE metal, pure aluminum, the
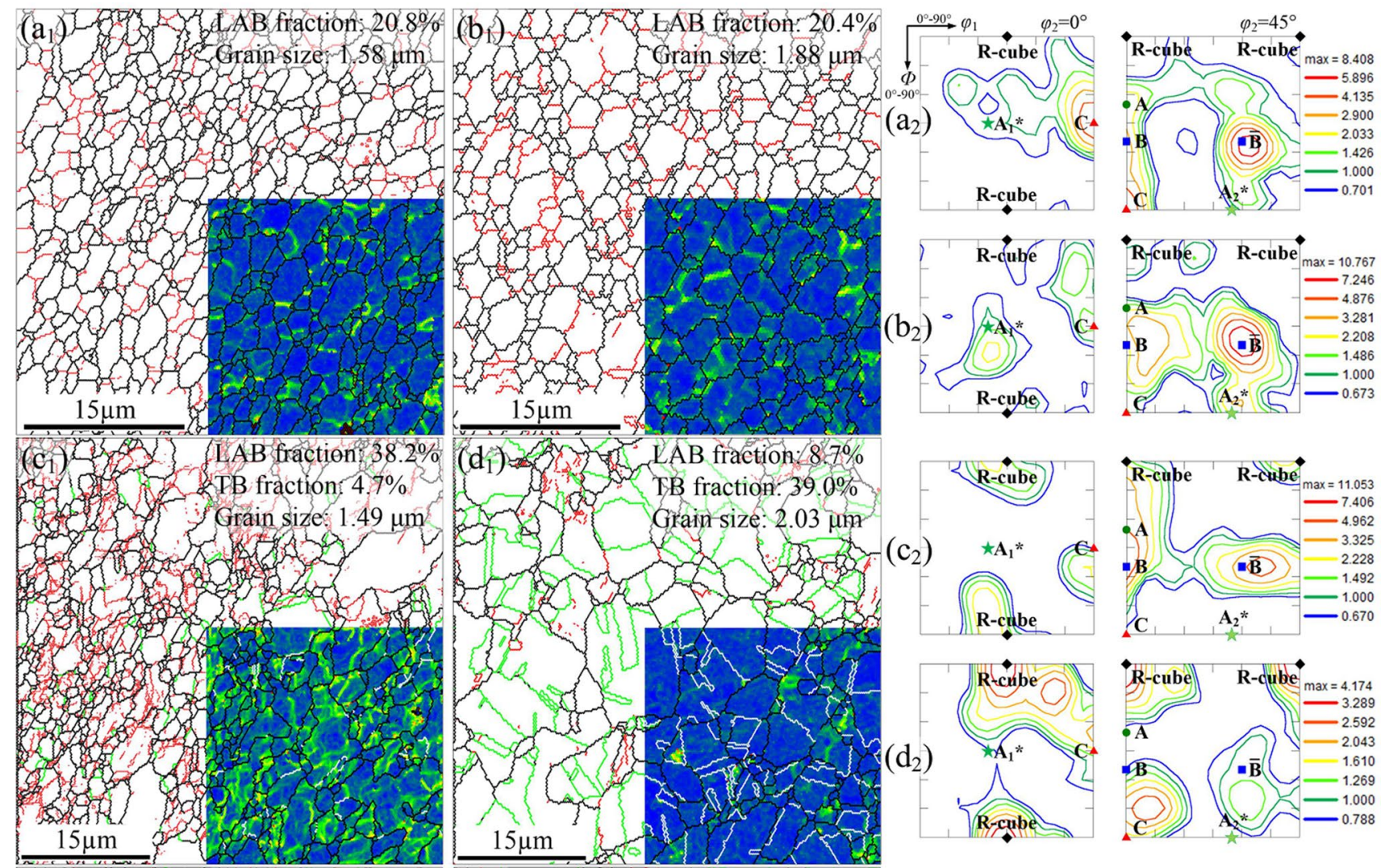

$\left(e_{1}\right)$ L L AB fraction: $18.9 \%$
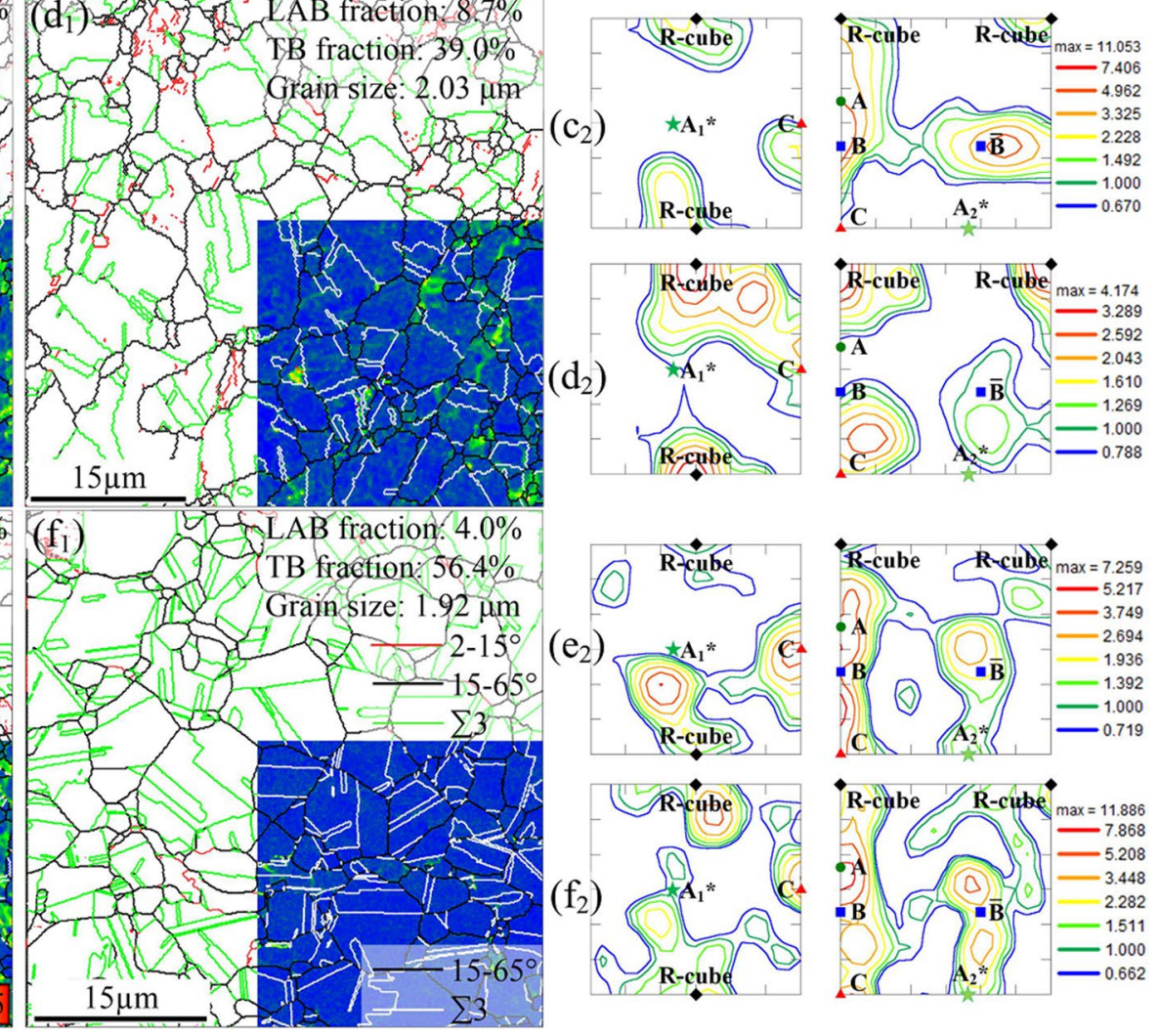

$\left(d_{2}\right)$
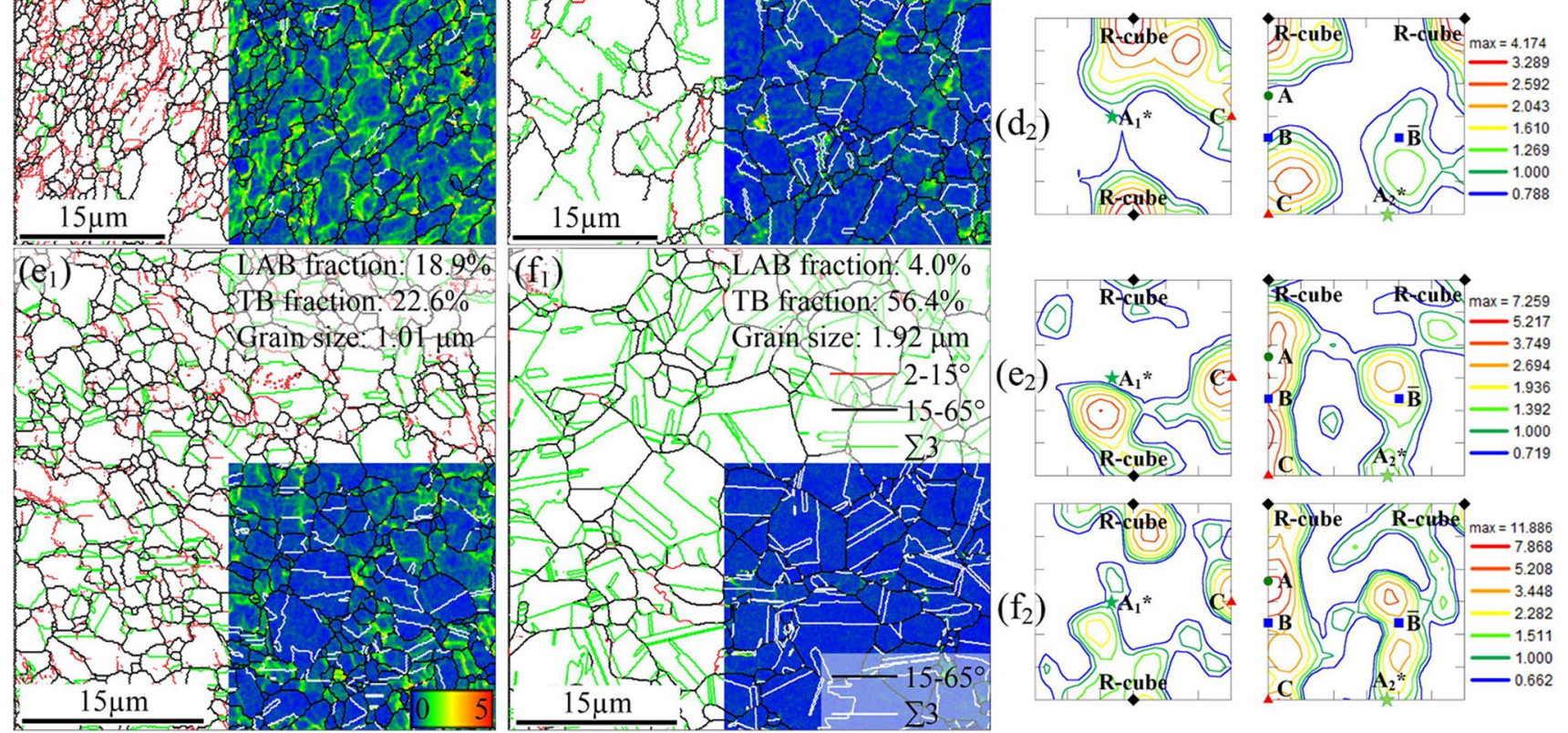

Fig. 7 Microstructural features at the material flow ending position (P4) before $\mathbf{a}_{\mathbf{1}}, \mathbf{c}_{\mathbf{1 , 2}}, \mathbf{e}_{\mathbf{1 , 2}}$ and after a short-time annealing $\mathbf{b}_{\mathbf{1 , 2}}, \mathbf{d}_{\mathbf{1 , 2}}, \mathbf{f}_{\mathbf{1 , 2}}: \mathbf{a}_{\mathbf{1}}-\mathbf{f}_{\mathbf{1}}$ EBSD grain boundary maps and KAM maps; $\mathbf{a}_{\mathbf{2}}-\mathbf{f}_{\mathbf{2}}$ the corresponding ODF maps; $\mathbf{a}_{\mathbf{1 , 2}}, \mathbf{b}_{\mathbf{1 , 2}}$ pure aluminum; $\mathbf{c}_{\mathbf{1 , 2}}, \mathbf{d}_{\mathbf{1 , 2}}$ pure copper; $\mathbf{e}_{\mathbf{1 , 2}}, \mathbf{f}_{\mathbf{1 , 2}} \mathrm{Cu}-$ $30 \mathrm{Zn}$ 
EBSD grain boundary map and the corresponding KAM map before and after a short-time annealing are shown in Fig. $7 a_{1}$ and $b_{1}$, respectively. The corresponding orientation distribution functions (ODFs) before and after the short-time annealing are shown in Fig. $7 a_{2}$ and $b_{2}$. The typical simple shear texture components and annealing texture (Rotatedcube texture) are also marked in the ODF maps [25]. They have been widely reported in the FSW of FCC metals [19, $21,22]$. Similar to those at the recrystallization beginning position and the high-speed flowing stage, the grain structure at the material flow ending position before the short-time annealing showed highly recrystallized features. The LAB fraction was only $20.8 \%$. The dislocation density was also very low. After the short-time annealing, the grain structure was nearly same except for the grain size increasing from $\sim 1.58$ to $1.88 \mu \mathrm{m}$. The ODF maps in Fig. $7 \mathrm{a}_{2}$ and $\mathrm{b}_{2}$ show that the $B / \bar{B}$ and $C$ components predominated the texture before the short-time annealing. After the short-time annealing, the intensity of the $B / \bar{B}$ components enhanced, while the intensity of the $C$ component weakened. This indicated that the preferred grain growth along the $B / \bar{B}\{112\}$ $\langle 110\rangle$ orientation occurred during the short-time annealing [27].

For the mid-SFE metal, pure copper, the grain structure at the material flow ending position before the short-time annealing was similar to that at the high-speed flowing stage. It consisted of a few recrystallized grains with $\sum 3$ TBs inside and a lot of deformed grains with a lot of LABs inside, as shown in Fig. $7 \mathrm{c}_{1}$. Also, the dislocation density was very high. After the short-time annealing, the LAB fraction decreased from 38.2 to $8.7 \%$, while the $\sum 3 \mathrm{~TB}$ fraction increased from 4.7 to $39 \%$. In addition, the dislocation density remarkably decreased, and the grain size increased from 1.49 to $2.03 \mu \mathrm{m}$, as shown in Fig. $7 \mathrm{~d}_{1}$. The ODF maps in Fig. $7 \mathrm{c}_{2}$ and $\mathrm{d}_{2}$ show that the $B / \bar{B}$ components predominated the texture before the short-time annealing. However, after the short-time annealing, the rotated cube texture (R-cube) turned into the dominant. The intensity of the $B / \bar{B}$ components significantly weakened. These results indicated that for the mid-SFE metal, the static recrystallization occurred during the short-time annealing [25].

For the low SFE metal, $\mathrm{Cu}-30 \mathrm{Zn}$, the grain structures at the material flow ending position before and after the short-time annealing are shown in Fig. $7 \mathrm{e}_{1}$ and $\mathrm{f}_{1}$. Before the short-time annealing, in contrast with that of the midSFE metal, the LAB fraction decreased remarkably, while the $\sum 3$ TB fraction increased significantly. The dislocation density was also lower than that of the mid-SFE metal. This can be attributed to the special grain structure evolution mechanism which occurred at the material high-speed flowing stage during the FSW of low SFE metal. After the short-time annealing, the grain size increased from 1.01 to $1.92 \mu \mathrm{m}$, accompanied by the LAB fraction decreasing and the $\sum 3 \mathrm{~TB}$ fraction increasing. This is similar to that of the mid-SFE metal. However, the ODF maps in Fig. $7 \mathrm{e}_{2}$ and $\mathrm{f}_{2}$ show that the texture types had no obvious change before and after the short-time annealing. Both before and after the short-time annealing, the $\langle 110\rangle$ fiber components dominated the texture, which was attributed to the simple shear deformation occurring during the material flow. The intensity of the rotated-cube texture was very weak all the time. These results indicated that no static recrystallization occurred during the short-time annealing for the low SFE metal. Only normal grain growth occurred in the short-time annealing during which a large number of annealing twins formed [29].

\section{Discussion}

The microstructure features of FCC metals with different SFE at different stages during the FSW have been presented in detail hereinbefore. Basically speaking, these features originated from the behaviors of the dislocation motion and the grain boundary migration during the plastic deformation in FSW. However, both the dislocation motion and the grain boundary migration were significantly influenced by the SFE. Therefore, the grain structure evolution was also highly dependent on the SFE. The SFE is a measure of the energy per unit area required when introducing stacking fault per unit area in a perfect crystal [41]. It affects the dislocation motion and grain boundary migration in two ways. On the one hand, in crystal materials, the full dislocations tend to dissociate into two or more partial dislocations and stacking fault (so called extended dislocations), reaching a lower energy and more stable state. The width of the extended dislocations significantly affects the dislocation cross-slip and dislocation climb. This is because the dislocation crossslip and dislocation climb can occur only when the extended dislocations shrink into full dislocations. However, the wider are the extended dislocations, the more difficultly the extended dislocations shrink into full dislocations. The relationship between the width of the extended dislocations and the SFE can be described by the following equation [41]: $d=\frac{G b_{2} b_{3}}{2 \pi \gamma_{\mathrm{SFE}}}$, where $d$ is the width of the extended dislocations, $G$ is the shear modulus, $\gamma_{\mathrm{SFE}}$ is the SFE, $b_{2}$ and $b_{3}$ are the burgers vector of the partial dislocations. It can be seen that the lower is the SFE, the wider is the extended dislocations, the more difficultly the dislocation cross-slip and dislocation climb occur. On the other hand, the lower is the SFE, the higher is the occurrence probability of stacking fault during the atom rearrangement, especially during the HAB migration. For the FCC metals, when an intrinsic-type stacking fault incidentally occurs, it actually forms a $\sum 3$ TB [38]. Therefore, a mass of annealing twin forms during the HAB migration of low SFE metals. 
The grain structure evolution during the FSW of FCC metals with different SFE under the typical welding parameters (welding peak temperature: $0.6 T_{\mathrm{m}}-0.7 T_{\mathrm{m}}$ ) is summarized and schematically shown in Fig. 8. At the deformation beginning stage, the deformation temperature is not very high. In the high SFE metals, owing to that the width of the extended dislocation is small, the dislocation cross-slip and dislocation climb occur easily. Therefore, the DRV occurs during the plastic deformation, forming a large number of subgrains [27]. In the mid-SFE metals, the occurrence probability of the dislocation cross-slip and dislocation climb significantly decreased, but they still occur by accident. A few of dislocation boundaries randomly form in the deformed grain in this way. These dislocation boundaries as well as the original grain boundary will become the obstacles to the dislocation movement. Thus, the dislocations are randomly accumulated in the deformed grain interior and boundaries [25]. Meanwhile, the $\sum 3$ TBs absorb the dislocations and transform into general HABs. For the low SFE metals, the dislocation cross-slip and dislocation climb hardly occur. In the grain interior, the dislocation boundary is very difficult to form. Only the original HABs and TBs are the obstacles to the dislocation movement. Therefore, a large number of dislocations are accumulated along the original HABs and the $\sum 3$ TBs [29]. Also, the $\sum 3$ TBs absorb the dislocations and transform into general HABs.

At the recrystallization beginning stage, the deformation temperature is relatively high. In the high SFE metals, the elevated temperature and continuous deformation lead to the CDRX. The continual dislocation accumulation and rearrangement result in the continual increasing of the misorientation angle, and thus, the LAB transforms into HAB [27]. For the mid-SFE metals, the random dislocation accumulation leads to an apparent difference in the dislocation density on opposite sides of the grain boundary in the local area, and thus, the HAB bulging occurs there. The HAB bulging leads to a low dislocation density region, which becomes the nucleus of the recrystallized grain [25]. This process is known as the DDRX [36]. In the low SFE metals, owing to the dislocation accumulation along the grain boundary, the DDRX occurs along the grain boundary, forming the typical necklace-like structure [29]. It is worth mentioning that both in mid- and low SFE metals, $\sum 3$ TBs form in these recrystallized grains developed by the DDRX. However, the occurrence probability of the $\sum 3$ TBs in low SFE metals is much higher than that in the mid-SFE metals.

At the material high-speed flowing stage, the grain structure evolution is in a dynamic equilibrium state. For the high SFE metals, the CDRX combined with grain orientation spreading occurs during this stage [27]. The grains are always equiaxed grains. The grain size is nearly constant. The dislocations produced by the plastic deformation are fully absorbed by the grain boundaries and thus lead to the grain orientation spreading [27]. In the mid-SFE metals, as the plastic deformation continues, the dislocations can be accumulated again in the recrystallized grain interior, and

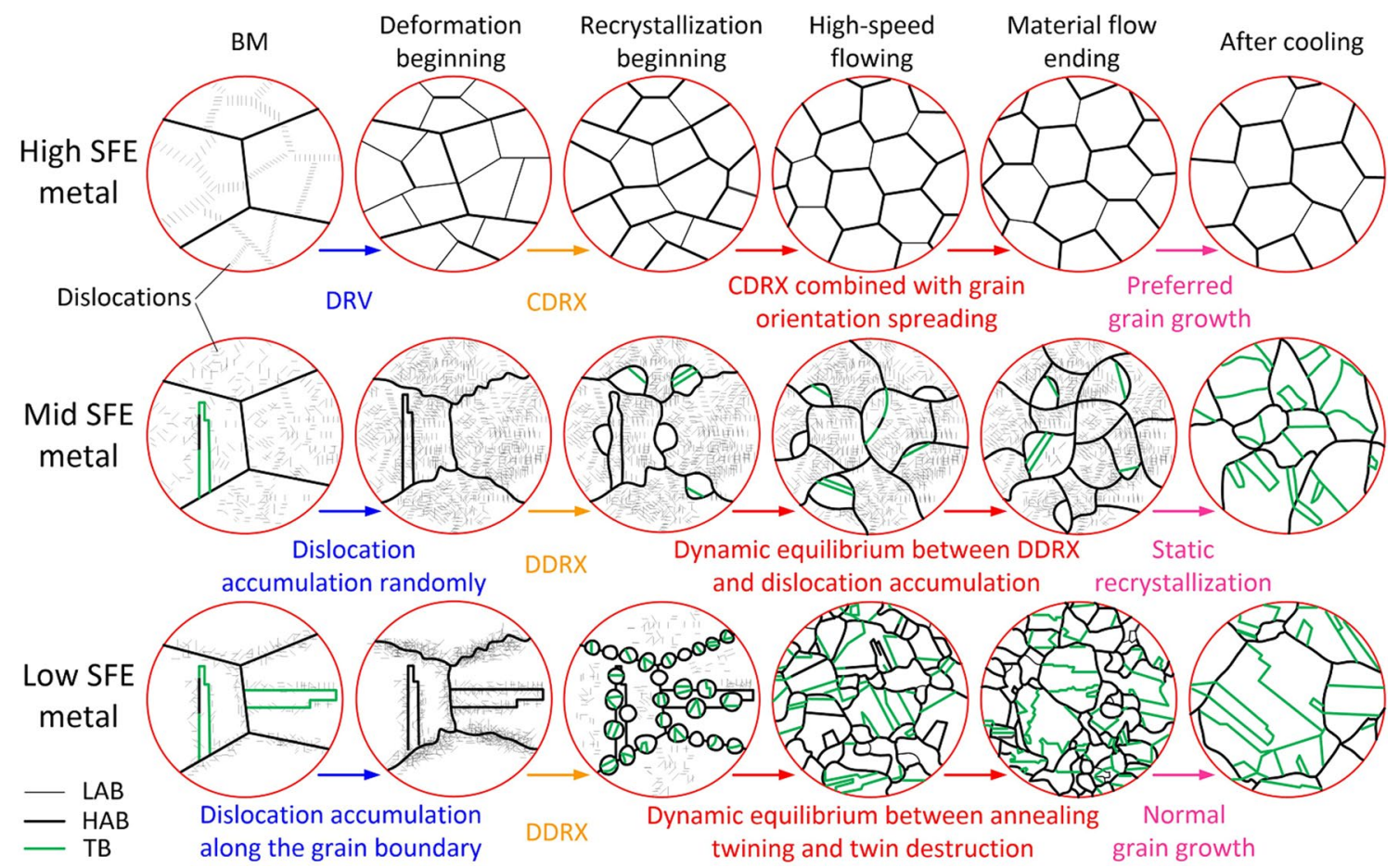

Fig. 8 Schematic of grain structure evolution during the FSW of metals with different SFE 
thus, the recrystallized grain becomes the deformed grain. The DDRX therefore occurs again in the new deformed grain. The grain structure evolution is in the dynamic equilibrium between the DDRX and the dislocation accumulation produced by the continuous deformation [25]. However, in the low SFE metals, on the one hand, the $\sum 3$ TBs form frequently during the thermally activated HABs migration. On the other hand, the dislocations are accumulated along the grain boundaries (including the $\sum 3 \mathrm{TBs}$ ). The $\sum 3 \mathrm{TBs}$ absorb the dislocations and transform into general HABs and thus accommodate the plastic deformation. The generated HABs continue to move under the thermal activation and give rise to the $\sum 3$ TBs again. Therefore, the grain structure evolution is in the dynamic equilibrium between the annealing twinning due to the thermally activated grain boundary migration and the twin destruction due to the continuous deformation [29].

At the cooling stage, the grain structure undergoes a short-time annealing. For the high SFE metals, the preferred grain growth occurs during this stage, resulting in the increase in grain size and the texture sharpening [27, 30]. For the mid-SFE metals, owing to many dislocations remaining at the end of the material flow, the static recrystallization occurs during the cooling stage [25]. After the cooling, the grain structure is characterized by the coarse grains and annealing twins. For the low SFE metals, not like the midSFE metals, the dynamic equilibrium between the annealing twinning due to the thermally activated grain boundary migration and the twin destruction due to the continuous deformation leads to few dislocations remaining at the end of the material flow. Because of this, the driving force for static recrystallization at the cooling stage is not enough. Only the normal grain growth occurs at the cooling stage [29].

\section{Conclusions}

This study summarized the grain structure evolution of metals with different SFE during the FSW under the conventional welding parameters (welding peak temperature: 0.6 $T_{\mathrm{m}}-0.7 T_{\mathrm{m}}$ ). The effect of the SFE on the grain structure evolution at different welding stages was revealed. The following conclusions can be drawn:

1. At the deformation beginning stage, as the SFE decreases, the DRV, the dislocation accumulation randomly in the grain interior and the dislocation accumulation along the original grain boundary occur successively.

2. At the recrystallization beginning stage, as the SFE decreases, the recrystallization mechanism changes from CDRX to DDRX.
3. At the material flow stage, as the SFE decreases, the grain structure evolution changes from the CDRX to DDRX, and further to the dynamic equilibrium between the annealing twinning due to thermally activated grain boundary migration and the twin destruction during the plastic deformation.

4. At the cooling stage, the static recrystallization occurs for the mid-SFE metals, while grain growth occurs for the high and low SFE metals.

Acknowledgements This study was partly financially supported by the New Energy and Industrial Technology Development Organization (NEDO) under the "Innovation Structural Materials Project (Future Pioneering Projects)" and a Grant-in-Aid for Science Research from the Japan Society for Promotion of Science. It was also financially supported by the China Postdoctoral Science Foundation funded Project (No. 2019M653726), the National Natural Science Foundation of China (No. 51905437) and the Fundamental Research Funds for the Central Universities (No. 3102019QD0407).

\section{References}

[1] W.M. Thomas, E.D. Nicholas, J.C. Needham, M.G. Murch, P. Templesmith, C.J. Dawes, Friction Stir Butt Welding, GB Patent 9125978.8, International Patent PCT/GB92/02203,1991

[2] G.K. Padhy, C.S. Wu, S. Gao, J. Mater. Sci. Technol. 34, 1 (2018)

[3] C.S. Wu, H. Su, L. Shi, Acta Metall. Sin. 54, 265 (2017). (in Chinese)

[4] G. Chen, S. Zhang, Y. Zhu, C. Yang, Q. Shi, Acta Metall. Sin. Engl. Lett. 33, 3 (2020)

[5] N. Xu, R.N. Feng, W.F. Guo, Q.N. Song, Y.F. Bao, Acta Metall. Sin. Engl. Lett. 33, 319 (2020)

[6] Z. Shen, Y. Ding, A.P. Gerlich, Crit. Rev. Solid State Mater. Sci. (2019). https://doi.org/10.1080/10408436.2019.1671799

[7] Y. Chen, H. Wang, X. Wang, H. Ding, J. Zhao, F. Zhang, Z. Ren, Mater. Sci. Eng. A 739, 272 (2019)

[8] Z.Y. Ma, A.H. Feng, D.L. Chen, J. Shen, Crit. Rev. Solid State Mater. Sci. 43, 269 (2018)

[9] B. He, L. Cui, D. Wang, H.J. Li, C.X. Liu, Acta Metall. Sin. Engl. Lett. 33, 135 (2020)

[10] R. Nandan, T. DebRoy, H.K.D.H. Bhadeshia, Prog. Mater. Sci. 53, 980 (2008)

[11] W.D. Callister, Fundamentals of Materials Science and Engineering (Wiley, London, 2000)

[12] F.J. Humphreys, M. Hatherly, Recrystallization and Related Annealing Phenomena (Elsevier, Amsterdam, 2012)

[13] P.B. Prangnell, C.P. Heason, Acta Mater. 53, 3179 (2005)

[14] S. Mironov, Y.S. Sato, H. Kokawa, Acta Mater. 56, 2602 (2008)

[15] S. Mironov, Y.S. Sato, H. Kokawa, Acta Mater. 57, 4519 (2009)

[16] W. Wang, P. Han, P. Peng, T. Zhang, Q. Liu, S.N. Yuan, L.Y. Huang, H.L. Yu, K. Qiao, K.S. Wang, Acta Metall. Sin. Engl. Lett. 33, 43 (2020)

[17] K.V. Jata, S.L. Semiatin, Scr. Mater. 8, 743 (2000)

[18] J.Q. Su, T.W. Nelson, C.J. Sterling, Mater. Sci. Eng. A 405, 277 (2005)

[19] R.W. Fonda, J.F. Bingert, K.J. Colligan, Scr. Mater. 51, 243 (2004)

[20] Y. Huang, Y. Xie, X. Meng, J. Li, Mater. Sci. Eng. A 740, 211 (2019)

[21] S. Mironov, K. Inagaki, Y.S. Sato, H. Kokawa, Philos. Mag. 95, 367 (2015) 
[22] S. Mironov, K. Inagaki, Y.S. Sato, H. Kokawa, Philos. Mag. 94, 3137 (2014)

[23] X.C. Liu, Y.F. Sun, Y. Morisada, H. Fujii, J. Mater. Process. Technol. 252, 643 (2018)

[24] X.C. Liu, Y.F. Sun, T. Nagira, K. Ushioda, H. Fujii, Sci. Technol. Weld. Join. 24, 352 (2019)

[25] X.C. Liu, Y.F. Sun, T. Nagira, K. Ushioda, H. Fujii, J. Mater. Sci. Technol. 35, 1412 (2019)

[26] X.C. Liu, Y.F. Sun, T. Nagira, K. Ushioda, H. Fujii, Materialia 6, 100302 (2019)

[27] X.C. Liu, Y.F. Sun, H. Fujii, Mater. Des. 129, 151 (2017)

[28] X.C. Liu, Y.F. Sun, T. Nagira, H. Fujii, Mater. Character. 137, 24 (2018)

[29] X.C. Liu, Y.F. Sun, T. Nagira, K. Ushioda, H. Fujii, J. Mater. Sci. 53, 10423 (2018)

[30] D. Yi, S. Mironov, Y.S. Sato, H. Kokawa, Philos. Mag. 96, 1965 (2016)
[31] T. Hasegawa, U.F. Kocks, Acta Metall. 27, 1705 (1979)

[32] H.J. McQueen, E. Evangelista, J. Phys. B 38, 359 (1988)

[33] S. Gourdet, F. Montheillet, Mater. Sci. Eng. A 283, 274 (2000)

[34] M.R. Barnett, F. Montheillet, Acta Mater. 50, 2285 (2002)

[35] S. Mironov, K. Inagaki, Y.S. Sato, H. Kokawa, Metall. Mater. Trans. A 46, 783 (2015)

[36] D.G. Cram, H.S. Zurob, Y.J.M. Brechet, C.R. Hutchinson, Acta Mater. 57, 5218 (2009)

[37] A. Heidarzadeh, T. Saeid, V. Klemm, A. Chabok, Y. Pei, Mater. Des. 162, 185 (2019)

[38] Y. Jin, Annealing Twin Formation Mechanism, Diss. Ecole ationale Supérieure des Mines de Paris (2014)

[39] N. Xu, R. Ueji, H. Fujii, J. Mater. Process. Technol. 232, 90 (2016)

[40] T. Nagira, X.C. Liu, K. Ushioda, Y. Iwamoto, G. Ano, H. Fujii, Sci. Technol. Weld. Join. 24, 644 (2019)

[41] J. Pelleg, Mechanical Properties of Materials (Springer, Beer Sheva, 2013) 